\title{
Article
}

\section{Review of isolation enhancement with the help of theory of characteristic modes}

\author{
Farhan Ammar Ahmad ${ }^{1, *}$ \\ 1 Department of Electrical Engineering, University of Management and Technology Lahore, Sialkot campus, Pakistan. \\ * Correspondence: fahran.sayal@post.umt.edu.pk; Tel.: +923234046006
}

Received: 14 December 2018; Accepted: 27 February 2019; Published: 3 March 2019.

\begin{abstract}
Multiple-input-multiple-output (MIMO) antennas performance can be degraded due to the poor isolation between the MIMO antenna elements. In this paper, we present a review of the different isolation enhancement schemes available in the literature. Empirically the isolation between the antennas can be improved by placing the antenna as far as possible and it can be enhanced further by introducing different isolation enhancement schemes. Theory of characteristic modes (TCM) was recently proposed that has useful benefits. TCM is also used to enhance the isolation. Moreover, this papers focus on the different approaches of TCM, to enhance the isolation.
\end{abstract}

Keywords: Antennas, defected ground structures (DGS), isolation enhancement, MIMO antennas, theory of characteristic modes (TCM).

\section{Introduction}

$\mathbf{T}$

heory of characteristic modes (TCM) was developed by Garbacez [1] and gained importance after it was revisited in [2] by diagonalizing the impedance matrix of the body. Mathematically, we have [3-6]

$$
[Z] I_{n}=\left(1+j \lambda_{n}\right)[R] I_{n}
$$

where $Z=R+j X, I_{n}$ and $1+j \lambda_{n}$ represents the impedance, current eigen vector and eigen value respectively. Further simplification of the (1) gives

$$
[X] I_{n}=\lambda_{n}[R] I_{n}
$$

It can also be represented as

$$
[X] J_{n}=\lambda_{n}[R] J_{n}
$$

Here $J_{n}$ represents the current density eigen vector. From the Theorem of Reciprocity, if $Z$ is linear symmetric operator then the Hermitian parts of $Z(R$ and $X)$ will also be linear symmetric operator [3]. Thus we concludes that the eigen values will be real and eigen vectors will be real and equiphasal. Eigen value gives us an information about the behavior of a modes at a particular frequency whether it will resonate or store electrical/mechanical energy. Modal significance (MS) is a parameter depending on the eigen value and gives us information about the contribution of a particular mode at a particular frequency. Mathematically it is given by

$$
M S_{n}=\left|\frac{1}{1+j \lambda_{n}}\right|
$$

At resonance, the value of MS is equal to 1 and at $3 \mathrm{~dB}$, the value of $\mathrm{BW}$ corresponds to 0.707 . The radiation pattern associated with these real eigen modes are orthogonal to one another.

TCM is widely used for the analysis of various different types of antennas such as wire antennas [7], handset antennas [8], small antennas [9], dielectric antennas [10], printed MIMO antennas [11-16] and slot antennas [17-19]

Multiple-input-multiple-output (MIMO) antennas are widely used for the enhancement of antenna capacity and this technology is used for $4 \mathrm{G}$ and $5 \mathrm{G}$ communications [20,21]. The performance of such antennas 
can be degraded, if the antennas are not properly isolated [21,22]. Recently TCM was used to enhance the isolation between the MIMO antenna elements.

The main contribution of this paper is to review the empirical method procedure (EMP) used for the isolation enhancement. We also review the isolation enhancement from the perspective of TCM. The short comings and the significance of the methods proposed in the literature with respect to TCM is highlighted. The breakup of this paper is: in section 2 we briefly discuss the different methods given in the literature from EMP method. In section 3 we discuss the isolation enhancement from the perspective of TCM. The conclusion is provided in section 4 .

\section{Isolation Enhancement using EMP}

From EMP perspective the isolation between MIMO antennas can be enhanced by the use of meta surfaces or electromagnetic bandgap structures [23-25], parasitic elements [26,27], neutralization line technique [28,29], optimization of the antenna system configuration [30,31], decoupling networks [32] and the use of Defected ground structures (DGS)[11,33-42]. Among all the aforementioned port isolation enhancement methods, DGS is the least complex and expensive.

Meta-material (single layer or double layer) are used for miniaturization and isolation. They suppress surface waves between the antennas resulting in an enhanced isolation [23-25]. Meta-materials normally involve larger and bulky formations that needs to be properly optimized. The isolation enhancement cant be obtained easily, if miniaturization is the major objective. Isolation improvement in [32] by the use of decoupling methods normally compromises of two stages: matching network and decoupling network. In decoupling network reactive elements are designed between the antenna feeding ports to improve the isolation, such networks require more space and generates additional cost. In addition, neutralization line technique was proposed in $[28,29]$. These lines deliver certain amount of signal to the neighboring antenna that counteracts for the coupling between them. The major drawback is huge amount of optimization involved in the adjustment of the width, length and the location of connection point of these lines. More than one neutralization line is needed to improve the isolation over wide frequency bandwidth and this complicates the process further. Parasitic elements also uses the idea of field cancellation to enhance the isolation between the antennas [26,27]. The major draw back is the optimization of the shape and the dimensions of parasitic element that play significant role in isolation enhancement.

In [33], a periodic $S$ shaped DGS was used to improve the isolation between two patch antennas to $23 d B$. Two DGS (T-shaped and line slot) were used to improve the isolation to $18 d B$ over a wide frequency range of 3.1-10.6 GHz in [34]. Two DGS were used to address two different frequency bands. A DGS etched in a square ring fashion was used to improve isolation by $7 d B$, for a square patch surrounded by a square ring patch design in [35]. An isolation was improved to $17 d B$ for a 2 element 4 shaped dual band design, where the length of the rectangular slot and spirals in the DGS were used to tune the frequency band in [36]. In [37], an isolation of $15 d B$ was achieved for a 2 element MIMO antenna by combining two isolation enhancement mechanisms that are orthogonal placement of antennas and introducing a slit in the ground. Isolation enhancement for a MIMO antenna system for more than 2 elements is difficult. Isolation was enhanced to $12 d B$ for a very closely packed 4-element MIMO design, where they used four wideband DGS with multi objective fractional factorial design [38]. A slitted pattern was etched between 2-element PIFA and wire monopole design that enhanced the isolation to reach $20 d B$ [39]. Moreover, the study was extended to 4-element PIFA (aligned along a line) to get an isolation of $12 d B$. Two DGS were used in [40] to improve isolation of a 4-element MIMO design. A rectangular slot and a stair case slot were used to enhance the isolation to $12 d B$ between the horizontal and verticals antennas respectively. For an 8-element MIMO design, a very complicated DGS consisting of closed loop frequency selective surfaces and quad strips connected with a circular arc were used to improve isolation to $15 d B$ respectively [42].

The main problem in the DGS method is to enhance isolation is the shape, size, number of DGS, position and the huge amount of optimization involved in the placement of DGS. We observed that from the perspective of EMP there is no systematic method to enhance the isolation. All of the available literature rely on the past experience and different parametric studies to obtain the enhanced isolation. This was the reason that the TCM was used for the isolation enhancement to develop a proper methodology for isolation enhancement. 


\section{Isolation Enhancement using TCM}

The presence of any antenna or deformation in the chassis greatly affect the chassis modes [43-47]. TCM was used in [48-52] to enhance the isolation between the MIMO antenna designs. It was demonstrated in [48] that the current in PIFA is more localized as compared Monopole and this is the reason that PIFA antennas are narrow band as compared to Monopole antennas. At the same time MIMO PIFA antenna design has better isolation because of its localized current nature. For frequencies less than $1 \mathrm{GHz}$, the chassis starts contributing to the antenna performance because now the electrical length of the antenna is huge, whereas for MIMO antennas, the situation will worsen because now all the antenna will excite the chassis modes. For frequency of $1 \mathrm{GHz}$, the antenna having only one chassis mode have electric field maxima at the edge and electric field minima at the center. An electrical antenna placed at the edge will effectively excite the chassis modes while antenna placed at the center will not. Antennas placed at the mentioned position achieved a $5 \mathrm{~dB}$ more isolation as compared to the antennas placed at the edges of the chassis [48]. A magnetic and electrical antenna placed at the edges will have improved isolation because the electrical antenna will excite chassis currents while the magnetic antenna will not [49]. Co-located antennas were introduced in [49] to have very compact MIMO antenna design. To have better isolation, the co-located Magnetic and electric antenna excite the chassis currents in opposite direction. In [48,49], only the behavior of the first mode using TCM was observed and analysis were based on it.

The selective excitation of characteristic modes that have orthogonal behavior can enhance the isolation. In [53], a feed network (consisting of 4 hybrid 180 couplers) and 4 capacitive coupler were designed to excite the four different modes of the antenna. The modes excited due to its orthogonal nature results in an enhanced isolation. The asymmetry in the ground plane produces a natural tilt and increase in directivity [54]. Two antennas were placed at asymmetrical ground plane such that both of them excite different characteristic modes, it will help in getting highly isolated and highly uncorrelated beams.

In [55], the lower order modes were separated by the use of decoupling network and GA was used to synthesize low quality factor MIMO antenna and quality factor is inversely proportional to the antenna bandwidth. A monopole (excited via CCE) and chassis (excited via ICE) combination was used to implement highly isolated MIMO design [56]. From the combination of CM, a new set of radiation modes can be formed, then the radiation modes are highly orthogonal to one another and thus resulting in highly uncorrelated beams [57].

In [58], out-of-band interference was improved by the use of TCM. For communication in aeroplane, antenna at 2.8 to $24 \mathrm{MHz}$ is used with very strong power. The harmonics of this antenna are high power and thus affects the communication of other antennas. Such type of interference is called out-of-band interference. The methodology adopted was to calculate the modal mutual admittance (MMA) and modal self admittance of the antenna. The CMA of the higher frequency antenna (with low power) is calculated. Functional and non-functional modes of the first antenna is calculated where modes contributing to real communication are known as functional while the modes contributing to interference are known as non-functional and non-functional modes are blocked by inductor loading with the help of TCM. The value of the inductor shall be properly optimized to block the non-functional mode.

In [59], designs made with the application of TCM were compared with the empirically made designs. 5 designs were opted from different papers with 2 designs opted from TCM and 3 opted by empirical method. All of the selected designs were MIMO and they were compared in 7 different real time scenarios. The scenarios were: first of all, the designs performance was observed in the presence of a box, where 3 scenarios in which the antenna was hold by one hand and the 3 scenarios in which the antenna was hold by two hands. TCM design recorded 3 times high multiplexing efficiency as compared to conventional design and for all the 7 scenarios, TCM designs performed with ME of $1.6 \mathrm{~dB}$ better.

Now all these methods focus on the isolation enhancement for frequentness around $1 \mathrm{GHz}[48,49]$. For a normal chassis, only one chassis mode of $1 \mathrm{GHz}$ is present. The problem will escalate, if we consider frequency greater than $1.5 \mathrm{GHz}$ as now more than one mode will be present. As the point where one mode was having current minima (a possible location for the placement of the second antenna), another mode current maxima lies over there. Thus we are left with no possible location.

In [12], a possible solution to this problem was proposed, where the DGS was used to enhance the isolation between two MIMO antennas. A methodology was also proposed to predict whether the isolation can be 
enhanced or not. The block diagram of the methodology is shown in the Figure 1. After the designing of MIMO antennas the characteristic modes were identified. The modes were classified as coupling and non-coupling modes based on the current distribution and its resemblance with the chassis current distribution in the presence of the excitation sources. Non-coupling modes are the one contributing to the radiation while the coupling modes are contributing to the port coupling thus degrading the isolation performance. If there exists a certain location on the chassis, where the DGS placed can block the coupling mode but at the same time does not effect the non-coupling mode the isolation between the antenna elements can be enhanced but if there does not exist any such location, the isolation between the antenna elements cannot be enhanced. The method was applied to different antenna designs and it achieved the most significant enhancement in the isolation as compared to other designs in the literature.

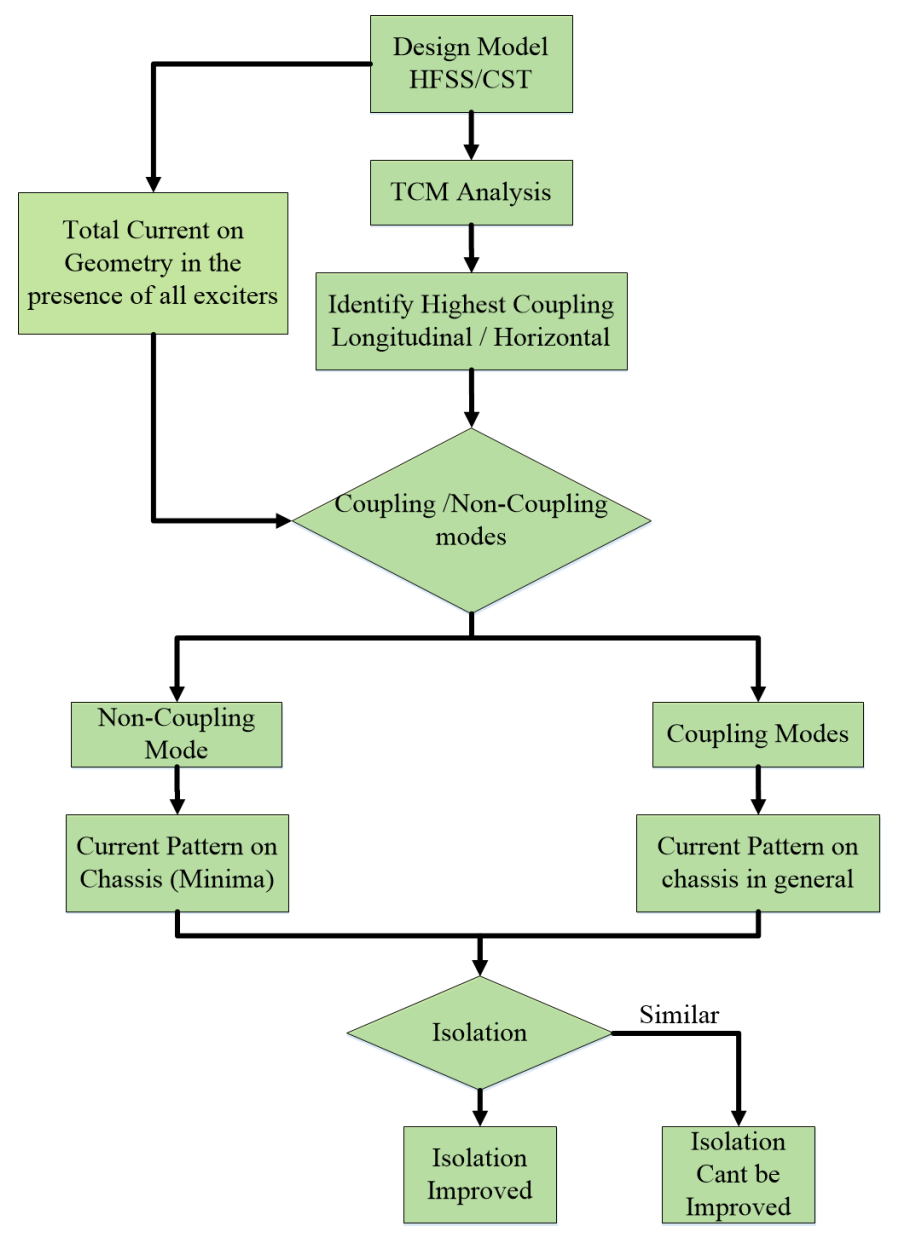

Figure 1. Block diagram of proposed isolation enhancement scheme that can predict whether the isolation can be enhanced or not [12]

\section{Conclusion}

In this paper, a summary of different available techniques to enhance the isolation between MIMO antenna elements is presented. The shortcomings of the different EMP based approaches and the TCM based approaches to enhance the port isolation between the MIMO antenna elements are discussed. A new method to enhance the isolation between the MIMO antennas with the help of TCM is proposed. The method is able to predict whether the isolation can be enhanced or not.

Conflicts of Interest: “The author declare no conflict of interest."

\section{References}


[1] Garbacz, R., \& Turpin, R. (1971). A generalized expansion for radiated and scattered fields. IEEE Transactions on Antennas and Propagation, 19(3), 348-358.

[2] Harrington, R., \& Mautz, J. (1971). Theory of characteristic modes for conducting bodies. IEEE Transactions on Antennas and Propagation, 19(5), 622-628.

[3] Cabedo Fabres, M. (2007). Systematic design of antennas using the theory of characteristic modes (Doctoral dissertation, Universidad PolitÃl'cnica de Valencia, ValÃíncia, Spain). Retrieved from https:/ / riunet.upv.es/bitstream/handle/10251/1883/tesisUPV2594.pdf?sequence=1

[4] Antonino Daviu, E. (2008). Analysis and design of antennas for wireless communications using modal methods (Doctoral dissertation, Universidad PolitÃl'cnica de Valencia, ValẤlncia, Spain). Retrieved from https:/ / riunet.upv.es/bitstream/handle/10251/2188/tesisUPV2809.pdf?sequence=1

[5] Ghalib, A. (2018). Current Engineering Methods Applied to the Design of MIMO Antennas (Unpublished doctoral dissertation), King Fahd University of Petroleum and Minerals (KFUPM), Dhahran, Kingdom of Saudi Arabia.

[6] Ghalib, A., \& Sharawi, M. S. (2016, September). A comparison between the antenna current green function and theory of characteristic modes. In 2016 IEEE Middle East Conference on Antennas and Propagation (MECAP) (pp. 1-4). IEEE.

[7] Cabedo-Fabres, M., Valero-Nogueira, A., \& Ferrando-Bataller, M. (2002, June). Systematic study of elliptical loop antennas using characteristic modes. In IEEE Antennas and Propagation Society International Symposium (IEEE Cat. No. 02CH37313) (Vol. 1, pp. 156-159). IEEE.

[8] Ethier, J., Lanoue, E., \& McNamara, D. (2008). MIMO handheld antenna design approach using characteristic mode concepts. Microwave and Optical Technology Letters, 50(7), 1724-1727.

[9] Newman, E. (1979). Small antenna location synthesis using characteristic modes. IEEE Transactions on Antennas and Propagation, 27(4), 530-531.

[10] Harrington, R., Mautz, J., \& Chang, Y. (1972). Characteristic modes for dielectric and magnetic bodies. IEEE Transactions on Antennas and Propagation, 20(2), 194-198.

[11] Ikram, M., Hussain, R., Ghalib, A., \& Sharawi, M. S. (2016, June). Compact 4-element MIMO antenna with isolation enhancement for 4G LTE terminals. In 2016 IEEE International Symposium on Antennas and Propagation (APSURSI) (pp. 535-536). IEEE.

[12] Ghalib, A., \& Sharawi, M. S. (2017). TCM analysis of defected ground structures for MIMO antenna designs in mobile terminals. IEEE Access, 5, 19680-19692.

[13] Ghalib, A., (2018). Theory of Characteristic modes application to MIMO Antennas. Forum for Electromagnetic research methods and application Technologies, 29.

[14] Ghalib, A., \& Sharawi, M. S. (2019). New antenna mode generation based on theory of characteristic modes. International Journal of RF and Microwave Computer-Aided Engineering, 29(3), e21686.

[15] Ghalib, A., \& Sharawi, M. S. (2016, September). Analyzing DGS behavior for a MIMO antenna system using theory of characteristic modes. In 2016 IEEE Middle East Conference on Antennas and Propagation (MECAP) (pp. 1-4). IEEE.

[16] Hussain, R., Ghalib, A., \& Sharawi, M. S. (2017). Annular slot-based miniaturized frequency-agile MIMO antenna system. IEEE Antennas and Wireless Propagation Letters, 16, 2489-2492.

[17] Ghalib, A., Hussain, R., \& Sharawi, M. S. (2018). Analysis of slot-based radiators using TCM and its application in MIMO antennas. International Journal of RF and Microwave Computer-Aided Engineering, 29(2), e21544.

[18] Ghalib, A., Hussain, R., \& Sharawi, M. S. (2017, July). Characteristic modes of circular slot antennas etched on a finite ground plane. In 2017 IEEE International Symposium on Antennas and Propagation E USNC/URSI National Radio Science Meeting (pp. 167-168). IEEE.

[19] Ghalib, A., Hussain, R., \& Sharawi, M. S. (2017, March). Low profile frequency agile MIMO slot antenna with TCM characterization. In 2017 11th European Conference on Antennas and Propagation (EUCAP) (pp. 2652-2655). IEEE.

[20] Ghalib, A., Sharawi, M. S., Attia, H., \& Mittra, R. (2018, August). Broadband Substrate Integrated Waveguide Slotted Array Antenna at mm-Wave Bands. In 2018 IEEE MTT-S International Microwave Workshop Series on 5G Hardware and System Technologies (IMWS-5G) (pp. 1-3). IEEE.

[21] Sharawi, M. S. (2014). Printed MIMO antenna engineering. Norwood, Massachusetts: Artech House.

[22] Ghalib, A., Clauzier, S., Sharawi, M. S., \& Antar, Y. M. (2016, June). A slotted waveguide based MIMO antenna system for wireless access points. In 2016 IEEE International Symposium on Antennas and Propagation (APSURSI) (pp. 1459-1460). IEEE.

[23] Zhai, G., Chen, Z. N., \& Qing, X. (2015). Enhanced isolation of a closely spaced four-element MIMO antenna system using metamaterial mushroom. IEEE Transactions on Antennas and Propagation, 63(8), 3362-3370.

[24] Ntaikos, D. K., \& Yioultsis, T. V. (2013). Compact split-ring resonator-loaded multiple-input-multiple-output antenna with electrically small elements and reduced mutual coupling. IET Microwaves, Antennas E Propagation, 7(6), $421-429$. 
[25] Hsu, C. C., Lin, K. H., \& Su, H. L. (2011). Implementation of broadband isolator using metamaterial-inspired resonators and a T-shaped branch for MIMO antennas. IEEE Transactions on Antennas and Propagation, 59(10), 3936-3939.

[26] Soltani, S., \& Murch, R. D. (2015). A compact planar printed MIMO antenna design. IEEE Transactions on Antennas and Propagation, 63(3), 1140-1149.

[27] Mak, A. C., Rowell, C. R., \& Murch, R. D. (2008). Isolation enhancement between two closely packed antennas. IEEE Transactions on Antennas and Propagation, 56(11), 3411-3419.

[28] Wang, Y., \& Du, Z. (2014). A wideband printed dual-antenna with three neutralization lines for mobile terminals. IEEE Transactions on Antennas and Propagation, 62(3), 1495-1500.

[29] Diallo, A., Luxey, C., Le Thuc, P., Staraj, R., \& Kossiavas, G. (2006). Study and reduction of the mutual coupling between two mobile phone PIFAs operating in the DCS1800 and UMTS bands. IEEE Transactions on Antennas and Propagation, 54(11), 3063-3074.

[30] Ramirez, R. R., \& De Flaviis, F. (2003). A mutual coupling study of linear and circular polarized microstrip antennas for diversity wireless systems. IEEE Transactions on Antennas and Propagation, 51(2), 238-248.

[31] Wu, B., \& Luk, K. M. (2011). A 4-port diversity antenna with high isolation for mobile communications. IEEE Transactions on Antennas and Propagation, 59(5), 1660-1667.

[32] Lui, C. Y., Wang, Y. S., \& Chung, S. J. (2008, July). Two nearby dual-band antennas with high port isolation. In 2008 IEEE Antennas and Propagation Society International Symposium (pp. 1-4). IEEE.

[33] Wei, K., Li, J., Wang, L., Xing, Z., \& Xu, R. (2016). S-shaped periodic defected ground structures to reduce microstrip antenna array mutual coupling. Electronics Letters, 52(15), 1288-1290.

[34] Luo, C. M., Hong, J. S., \& Zhong, L. L. (2015). Isolation enhancement of a very compact UWB-MIMO slot antenna with two defected ground structures. IEEE Antennas and Wireless Propagation Letters, 14, 1766-1769.

[35] Anitha, R., Sarin, V. P., Mohanan, P., \& Vasudevan, K. (2014). Enhanced isolation with defected ground structure in MIMO antenna. Electronics letters, 50(24), 1784-1786.

[36] Sharawi, M. S., Numan, A. B., Khan, M. U., \& Aloi, D. N. (2012). A Dual-Element Dual-Band MIMO antenna system with enhanced isolation for mobile terminals. IEEE antennas and wireless propagation letters, 11, 1006-1009.

[37] Ren, J., Hu, W., Yin, Y., \& Fan, R. (2014). Compact printed MIMO antenna for UWB applications. IEEE Antennas and Wireless Propagation Letters, 13, 1517-1520.

[38] Chen, Y. S., \& Chang, C. P. (2016). Design of a four-element multiple-input-multiple-output antenna for compact long-term evolution small-cell base stations. IET Microwaves, Antennas E Propagation, 10(4), 385-392.

[39] Chiu, C. Y., Cheng, C. H., Murch, R. D., \& Rowell, C. R. (2007). Reduction of mutual coupling between closely-packed antenna elements. IEEE Transactions on Antennas and Propagation, 55(6), 1732-1738.

[40] Hussain, R., \& Sharawi, M. S. (2016). Planar meandered-F-shaped 4-element reconfigurable multiple-input-multiple-output antenna system with isolation enhancement for cognitive radio platforms. IET Microwaves, Antennas \& Propagation, 10(1), 45-52.

[41] Ikram, M., Hussain, R., Hammi, O., \& Sharawi, M. S. (2016). An L-shaped 4-element monopole MIMO antenna system with enhanced isolation for mobile applications. Microwave and Optical Technology Letters, 58(11), $2587-2591$.

[42] Saleem, R., Bilal, M., Bajwa, K. B., \& Shafique, M. F. (2015). Eight-element UWB-MIMO array with three distinct isolation mechanisms. Electronics Letters, 51(4), 311-313.

[43] Ghalib, A., \& Sharawi, M. S. (2018). Analyzing antenna effects on mobile chassis currents using theory of characteristic modes. Microwave and Optical Technology Letters, 60(8), 1898-1905.

[44] Ghalib, A., \& Sharawi, M. S. (2017, March). Effects of actual antenna excitation on natural radiation modes. In 2017 11th European Conference on Antennas and Propagation (EUCAP) (pp. 3467-3470). IEEE.

[45] Ghalib, A., \& Sharawi, M. S. (2018, July). MIMO Antenna elements effect on chassis Modes. In 2018 IEEE International Symposium on Antennas and Propagation E USNC/URSI National Radio Science Meeting (pp. 1529-1530). IEEE.

[46] Ghalib, A., \& Sharawi, M. S. (2018, July). Effect of Antenna element placement on chassis Modes. In 2018 IEEE International Symposium on Antennas and Propagation \& USNC/URSI National Radio Science Meeting (pp. 103-104). IEEE.

[47] Ghalib, A., \& Sharawi, M. S. (2017, July). Excitation shape and placement effects on natural radiating modes. In 2017 IEEE International Symposium on Antennas and Propagation \& USNC/URSI National Radio Science Meeting (pp. 165-166). IEEE.

[48] Li, H., Tan, Y., Lau, B. K., Ying, Z., \& He, S. (2012). Characteristic mode based tradeoff analysis of antenna-chassis interactions for multiple antenna terminals. IEEE Transactions on Antennas and Propagation, 60(2), 490-502.

[49] Li, H., Lau, B. K., Ying, Z., \& He, S. (2012). Decoupling of multiple antennas in terminals with chassis excitation using polarization diversity, angle diversity and current control. IEEE Transactions on Antennas and Propagation, 60(12), 5947-5957. 
[50] Li, H., Xiong, J., Ying, Z., \& He, S. L. (2010). Compact and low profile co-located MIMO antenna structure with polarisation diversity and high port isolation. Electronics letters, 46(2), 108-110.

[51] Li, H., Lau, B. K., Tan, Y., He, S., \& Ying, Z. (2011, April). Impact of current localization on the performance of compact MIMO antennas. In Proceedings of the 5th European Conference on Antennas and Propagation (EUCAP) (pp. 2423-2426). IEEE.

[52] Li, H., Lau, B. K., \& Ying, Z. (2011, March). Optimal multiple antenna design for compact MIMO terminals with ground plane excitation. In 2011 International Workshop on Antenna Technology (iWAT) (pp. 218-221). IEEE.

[53] Chaudhury, S. K., Schroeder, W. L., \& Chaloupka, H. J. (2007, March). MIMO antenna system based on orthogonality of the characteristic modes of a mobile device. In 2007 2nd International ITG Conference on Antennas (pp. 58-62). IEEE.

[54] Szini, I., Tatomirescu, A., \& Pedersen, G. F. (2015). On small terminal MIMO antennas, harmonizing characteristic modes with ground plane geometry. IEEE Transactions on Antennas and Propagation, 63(4), 1487-1497.

[55] Yang, B., \& Adams, J. J. (2016). Systematic shape optimization of symmetric MIMO antennas using characteristic modes. IEEE Transactions on Antennas and Propagation, 64(7), 2668-2678.

[56] Martens, R., Holopainen, J., Safin, E., Ilvonen, J., \& Manteuffel, D. (2013). Optimal dual-antenna design in a small terminal multiantenna system. IEEE Antennas and Wireless Propagation Letters, 12, 1700-1703.

[57] Bouezzeddine, M., \& Schroeder, W. L. (2016). Design of a wideband, tunable four-port MIMO antenna system with high isolation based on the theory of characteristic modes. IEEE Transactions on Antennas and Propagation, 64(7), 2679-2688.

[58] Wu, Q., Su, W., Li, Z., \& Su, D. (2016). Reduction in out-of-band antenna coupling using characteristic mode analysis. IEEE Transactions on Antennas and Propagation, 64(7), 2732-2742.

[59] Vasilev, I., \& Lau, B. K. (2016). On user effects in MIMO handset antennas designed using characteristic modes. IEEE Antennas and Wireless Propagation Letters, 15, 758-761. 